\title{
Channel Covariance Identification in FDD Massive MIMO Systems ${ }^{\dagger}$
}

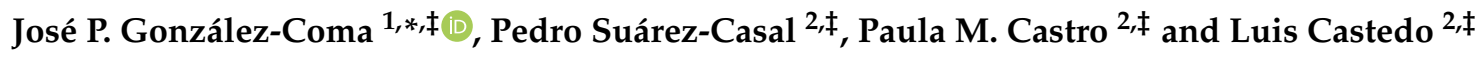 \\ 1 University of A Coruña, CITIC, A Coruña, Spain \\ 2 Department of Computer Engineering, University of A Coruña, A Coruña, Spain; \\ pedro.scasal@udc.es (P.S.-C.); paula.castro@udc.es (P.M.C.); luis.castedo@udc.es (L.C.) \\ * Correspondence: jose.gcoma@udc.es; Tel.: +34-881-016-051 \\ + Presented at the XoveTIC Congress, A Coruña, Spain, 27-28 September 2018. \\ $\ddagger$ These authors contributed equally to this work.
}

Published: 19 September 2018

check for updates

\begin{abstract}
Channel estimation for Massive MIMO systems has drawn a lot of attention in the last years. A number of estimation methods rely on the knowledge of the channel covariance matrix to operate. However, this covariance is not known in practice, and it should be acquired. In this work, we investigate different techniques for covariance identification under the assumption of very short training sequences.
\end{abstract}

Keywords: covariance identification; Massive MIMO; FDD

\section{Introduction}

Due to the large data rates necessary for future communication systems, Massive Multiple-Input Multiple-Output (MIMO) constitutes a promising candidate radio technology [1]. One of the features of this technology is the use of large antenna arrays. Therefore, large channel matrices have to be estimated at the user end in Frequency-Division Duplex (FDD) mode. Specifically, channel estimation is usually achieved by transmitting training sequences known to the transmitter and the receiver whose length, on the one hand, must be sufficient to obtain accurate estimates and, on the other, should be as short as possible to not impair data transmission efficiency. These circumstances make channel estimation a difficult task.

In recent literature, some authors consider covariance identification based on the assumption of channel reciprocity between the downlink and the uplink [2]. Moreover, [3,4] consider the FDD case by employing angular reciprocity instead.

\section{Materials and Methods}

In this work, we will focus on channel covariance identification for FDD on a more general scenario, that is, we assume that the channel is a stationary process whose statistics remain constant during a certain period of time. In addition, typical antenna arrangements like Uniform Linear Arrays (ULA) lead to covariance matrices with Toeplitz structure.

MUltiple SIgnal Classification (MUSIC) is a well-known Angle of Departure (AoD) estimation algorithm. For the considered problem, the MUSIC algorithm identifies the angles corresponding to the channel propagation paths. Thanks to the Toeplitz structure of the covariance matrix, we circumvent the typical limitations of MUSIC when the associated channel gain variances have to be estimated. Further, when the rank of the sample covariance matrix is smaller than the number of propagation paths, an interesting scenario arises. To address channel covariance identification in this scenario, 
we resort to the technique Spatial smoothing by employing training sequences designed as sparse rulers [5]. We have improved the technique proposed in [6] by using all the available information.

\section{Results}

In this section, we present some numerical results obtained with the proposed algorithms, together with the comparison with previous methods in the literature.

The following setup is considered for the numerical experiments. The number of transmit antennas is 400 for the channel covariance model of a ULA, and different number of channel paths. The training sequence was generated as a sparse ruler of length 50 . The methods evaluated are as follows:

- Covariance Orthogonal Matching Pursuit (COMP) [7]

- MUSIC

- $\quad$ Spatial Smoothing (SS)

- Improved Spatial Smoothing (ISS)

- $\quad$ Maximum Likelihood (ML) [8]

The performance metric is the Normalized Mean Squared Error (NMSE) between the actual channel covariance matrix and the estimated one. We try different levels of SNR, namely $0 \mathrm{~dB}$ and $30 \mathrm{~dB}$. The number of training periods indicates the number of channel block employed to estimate the channel covariance matrix, and might be smaller than the number of channel propagation paths.

NMSE vs. training periods for a moderate number of channel paths is shown in Figure 1a. Remarkably, MUSIC is more robust than COMP for the low SNR regime. ML is the best strategy in terms of performance but the algorithm is also the most computationally expensive, and may be not practical for realistic scenarios. Moreover, the other methods converge to ML when the number of snapshots is large enough. Regarding SS and ISS curves, we illustrate the gain of our approach with respect to the standard one.

In Figure $1 \mathrm{~b}$ the number of paths to estimate is much larger. MUSIC and COMP do not apply to this scenario due to the large number of parameters to be estimated. On the one hand, the restrictions of MUSIC regarding the sample covariance rank do not hold. On the other hand, COMP assumes sparsity, which does not apply in this setup. Therefore, we compare SS and ISS with ML that can be interpreted as a benchmark in the high SNR regime.

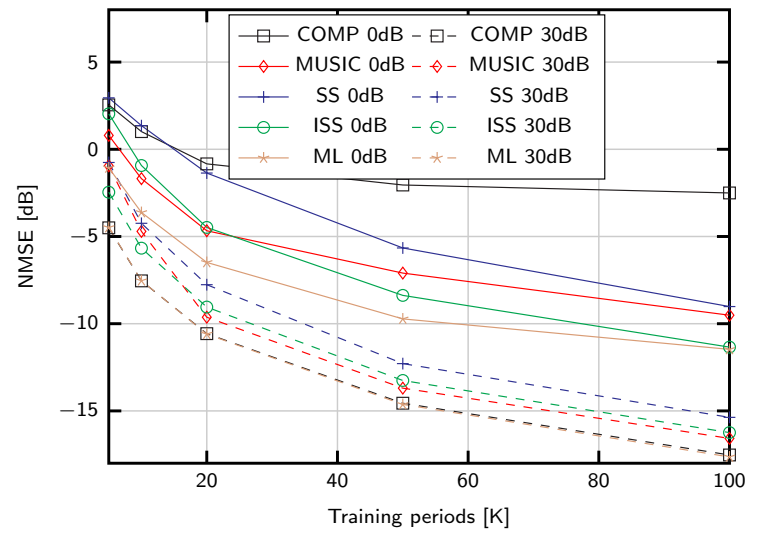

(a)

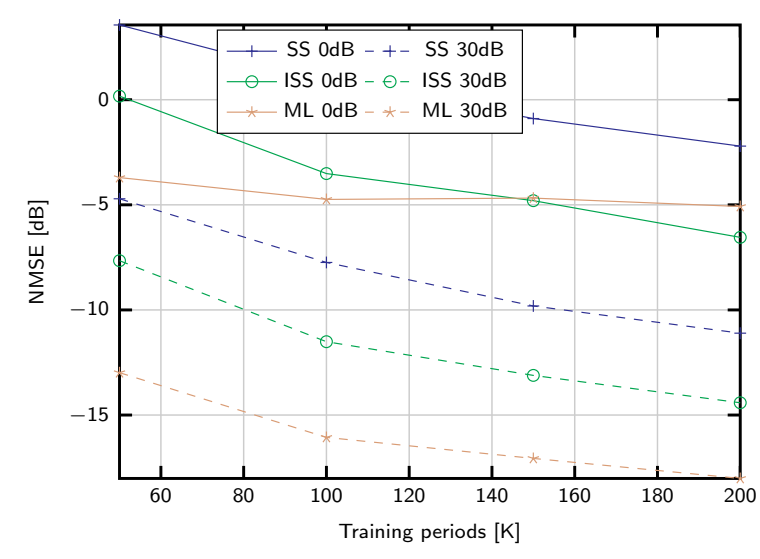

(b)

Figure 1. (a) NMSE of different covariance identification strategies for 400 antennas and 15 channel propagation paths. (b) NMSE of different covariance identification strategies for 400 antennas and 70 channel propagation paths. 


\section{Conclusions}

We have analyzed methods to identify the covariance matrix even if the sample covariance matrix is rank deficient. Moreover, we have updated the spatial smoothing method to improve the estimation quality in terms of NMSE.

Funding: This work has been funded by Xunta de Galicia (ED431C 2016- 045, ED341D R2016/012, ED431G/01), AEI of Spain (TEC2015-69648-REDC, TEC2016-75067-C4-1-R), and ERDF funds (AEI/FEDER, EU).

Conflicts of Interest: The authors declare no conflict of interest. The founding sponsors had no role in the design of the study; in the collection, analyses, or interpretation of data; in the writing of the manuscript, and in the decision to publish the results.

\section{References}

1. Rusek, F.; Persson, D.; Lau, B.K.; Larsson, E.G.; Marzetta, T.L.; Edfors, O.; Tufvesson, F. Scaling up MIMO: Opportunities and Challenges with Very Large Arrays. IEEE Signal Process. Mag. 2013, 30, 40-60, doi:10.1109/MSP.2011.2178495.

2. Neumann, D.; Joham, M.; Utschick, W. Covariance Matrix Estimation in Massive MIMO. IEEE Signal Process. Lett. 2018, 25, 863-867, doi:10.1109/LSP.2018.2827323.

3. Xie, H.; Gao, F.; Jin, S.; Fang, J.; Liang, Y. Channel Estimation for TDD/FDD Massive MIMO Systems with Channel Covariance Computing. arXiv 2017, arXiv:1710.00704.

4. Khalilsarai, M.B.; Haghighatshoar, S.; Yi, X.; Caire, G. FDD Massive MIMO via UL/DL Channel Covariance Extrapolation and Active Channel Sparsification. arXiv 2018, arXiv:1803.05754.

5. Romero, D.; Leus, G. Compressive covariance sampling. In Proceedings of the 2013 Information Theory and Applications Workshop (ITA), San Diego, CA, USA, 10-15 February 2013; pp. 1-8, doi:10.1109/ITA.2013.6502949.

6. Ariananda, D.D.; Leus, G. Direction of arrival estimation for more correlated sources than active sensors. Signal Process. 2013, 93, 3435-3448, doi:10.1016/j.sigpro.2013.04.011.

7. Park, S.; Heath, R.W., Jr. Spatial Channel Covariance Estimation for the Hybrid MIMO Architecture: A Compressive Sensing Based Approach. arXiv 2017, arXiv:1711.04207

8. Babu, P.; Stoica, P. Sparse spectral-line estimation for nonuniformly sampled multivariate time series: SPICE, LIKES and MSBL. In Proceedings of the 20th European Signal Process Conference (EUSIPCO), Bucharest, Romania, 27-31 August 2012; pp. 445-449.

(C) 2018 by the authors. Licensee MDPI, Basel, Switzerland. This article is an open access article distributed under the terms and conditions of the Creative Commons Attribution (CC BY) license (http:/ / creativecommons.org/licenses/by/4.0/). 